\title{
The very best limits on cosmological magnetic fields
}

\author{
Federico $R$ Urban $^{1, \star}$ \\ ${ }^{1}$ National Institute of Chemical Physics and Biophysics, Rävala 10 \\ 10143 Tallinn, Estonia
}

\begin{abstract}
I introduce you to the very best limits on cosmological magnetic fields. The numbers: magnetic fields coherent across a Jeans' length (around 2.4 Mpc today), are bound to be weaker than about $1 \mathrm{nG}(2 \sigma$ statement), whereas for a field as wide as the entire observable Universe the number is approximately $0.5 \mathrm{nG}$. These limits are obtained from rotation measures data, and as such they do not depend on the epoch at which the fields were generated.
\end{abstract}

\section{Why we care}

The Universe is swamped with all sorts of magnetic fields (MF): planetary MFs, galactic MFs, cluster MFs, possibly void MFs, etc. [1, 2]. Cosmological MFs (fields which imbue the entire Universe), on the other hand, remain elusive, but there is a whole world of physicists from different disciplines out there, ultra-high energy cosmic rays physics, structure formation, early and very early Universe theory, physics beyond the Standard Model, radio-astronomy, who would die to know more about them [3-5].

Cosmological MFs manifest themselves by rotating the plane of polarisation of electromagnetic waves propagating from far away sources to the Earth. Now, since we know that: (1) Faraday rotation measures (RM) of distant objects do not show any evolution with redshift $z$ [6], and (2) cosmological MFs (cosmoMF) generate RMs as we move away from here [7, 8], then (3) we can limit the strength of these cosmoMFs by comparing simulated and observed RMs distributions.

This contribution is based on my work with my collaborators Maxim Pshirkov and Peter Tinyakov [9].

\section{The new method}

\section{Observations.}

The only formula we need to know is that of Faraday rotation: the plane of polarisation of a linearly polarised electromagnetic wave which moves through a magnetised plasma rotates by an angle $\varphi$ proportional to the square of the wavelength $\lambda: \varphi=\operatorname{RM} \lambda^{2}$, where

$$
\mathrm{RM}=812 \int_{D}^{0} \frac{n_{\mathrm{e}}(z) B_{\|}(z)}{(1+z)^{2}}\left|\frac{\mathrm{d} l(z)}{\mathrm{d} z}\right| \mathrm{d} z .
$$

\footnotetext{
^e-mail: federico.urban@kbfi.ee
} 
Here $n_{\mathrm{e}}$ is the density of free electrons measured in $\mathrm{cm}^{-3}, B_{\|}$is the component of the MF (in $\mu \mathrm{G}$ ) parallel to the line of sight $l(z)$, and $D$ is the distance to the source in kpc; here and everywhere the $\mathrm{RM}$ is measured in $\mathrm{rad} / \mathrm{m}^{2}$.

What do we know about those RMs? First, the data: the largest set of RM of extragalactic sources to date was compiled in [10] from the NRAO VLA Sky Survey (NVSS) data [11]. The total number of observed sources was 37,543, of which 4002 have known redshifts [12]. These RMs consist of several contributions: we can split the observed $R M$ as $R_{\text {obs }}=R_{\text {gal }}+R R M$, where the first term comes from the regular MF of the Milky Way, and the second term stands for "residual RM": RM instrinsic to the source, measurement errors, turbulent galactic MF, and cosmoMF (xRRM) [13]. We massaged the data a bit to get the best grip on the redshift dependence, see [6] for all the tricks: after the massage we end up with a total of 2593 sources (our data points).

\section{Simulations.}

Like we said, if there is a cosmoMF the xRRM systematically grows with redshift due to its accumulation along the line of sight. To compute this we need $n_{\mathrm{e}}$, which we take from the observed Lyman- $\alpha$ forest distribution of neutral hydrogen absorption lines $[7,14,15]$. Next, since the conductivity of the Universe is extremely large, and since diffusion of the MF is inefficient at scales much larger than $1 \mathrm{AU}$, we can take a MF frozen into the plasma $[16,17]$. And then we run a simple code which spits out xRRM for a MF with strength $B$ and coherence length $l_{c}$, the latter ranging from a tenth of the Jeans length $\lambda_{J}$ to the Hubble size $1 / H_{0}$.

These simulations can generate only the contribution from the cosmoMF, but in real life there is a mickle of other stuff we do not (want to) know, so here is the stratagem: we exploit the data at low redshifts to obtain information about these unsung, redshift-independent, heroes, since there the cosmoMF is subdominant there, and use it together with our simulated XRRM at high redshift. In practice the algorithm is: (i) split the Universe in two redshift bands; (ii) make a first RM batch by randomly picking RRMs from the low- $z$ set; (iii) generate a second RM batch by simulating 100 xRRMs for each of the data sources of the high- $z$ set; (iv) blindly pluck one value from each of the batches and incoherently (that is, each with its own sign) add them a bunch of times to generate the final theoretical |RRM| distribution; (v) compare with actual data by means of a Kolmogorov-Smirnov test, which yields a p-value for the statistical (in)compatibility of the two distributions.

\section{The result}

See the self-explanatory Fig. 1.

\section{How we fare}

Currently, the strongest upper limits on the strength of present-day cosmoMFs come from microwave background observations [18] and read $B \lesssim 2.8 \mathrm{nG}$ for a coherence length $l_{c}=1 \mathrm{Mpc}$ - these limits apply only to primordial MFs, i.e., fields generated in the very early Universe. The limits coming RM data are looser [7, 19]: $\lambda_{J}$-scale fields are bound by $B \lesssim 6 \mathrm{nG}$. Our limits on the other hand read $1 \mathrm{nG}$ (this is a $2 \sigma$ statement) for $l_{c}=\lambda_{J}$, whereas $B \lesssim 0.5 \mathrm{nG}$ for the bloated field case, $l_{c}=1 / H_{0}$.

$$
\text { Conclusion: all in all, we win } \Theta
$$

\section{Acknowledgements.}

This work was supported by IISN project No. 4.4502.13 and Belgian Science Policy under IAP VII/37; my participation to QUARKS2016 was supported by ERC grant PUT808. 


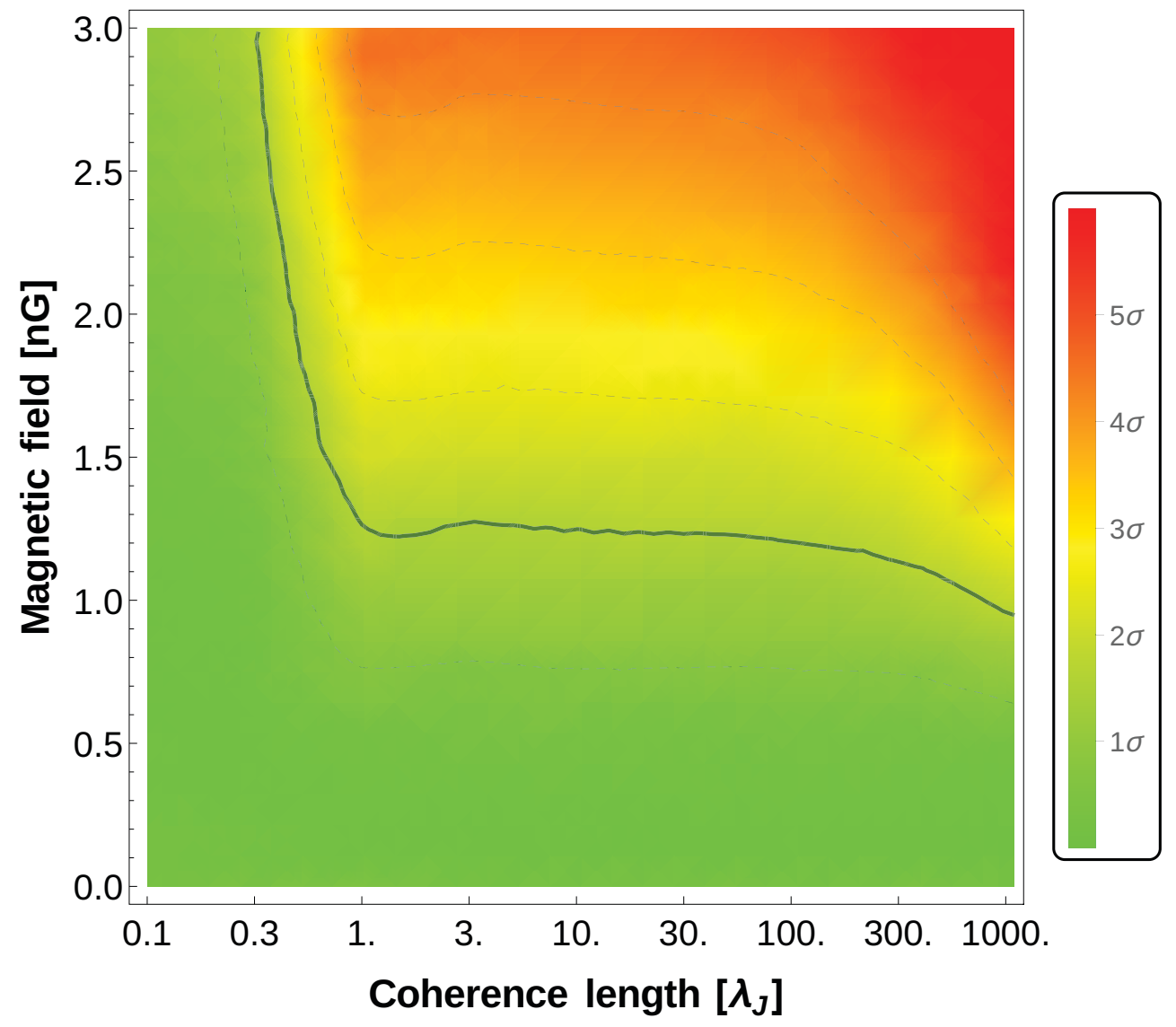

Figure 1. Confidence interval contours obtained from the p-values of the Kolmogorov-Smirnov tests as a function of the MF reference strength (y-axis) and coherence length (x-axis).

\section{References}

[1] J. P. Vallée, New Astron. Rev. 48 (2004) no.10, 763. doi:10.1016/j.newar.2004.03.017.

[2] D. Ryu, D. R. G. Schleicher, R. A. Treumann, C. G. Tsagas and L. M. Widrow, Space Sci. Rev. 166 (2012) 1 doi:10.1007/s11214-011-9839-z [arXiv:1109.4055 [astro-ph.CO]].

[3] O. Deligny, A. Letessier-Selvon and E. Parizot, Astropart. Phys. 21 (2004) 609 doi:10.1016/j.astropartphys.2004.04.012 [astro-ph/0303624].

[4] L. M. Widrow, D. Ryu, D. R. G. Schleicher, K. Subramanian, C. G. Tsagas and R. A. Treumann, Space Sci. Rev. 166 (2012) 37 doi:10.1007/s11214-011-9833-5 [arXiv:1109.4052 [astro-ph.CO]].

[5] R. Durrer and A. Neronov, Astron. Astrophys. Rev. 21 (2013) 62 doi:10.1007/s00159-013-0062-7 [arXiv:1303.7121 [astro-ph.CO]].

[6] M. S. Pshirkov, P. G. Tinyakov and F. R. Urban, Mon. Not. Roy. Astron. Soc. 452 (2015) 2851 doi:10.1093/mnras/stv1273 [arXiv:1407.3909 [astro-ph.GA]]. 
[7] P. Blasi, S. Burles and A. V. Olinto, Astrophys. J. 514 (1999) L79 doi:10.1086/311958 [astro$\mathrm{ph} / 9812487]$.

[8] T. Akahori and D. Ryu, Astrophys. J. 738 (2011) 134 doi:10.1088/0004-637X/738/2/134 [arXiv:1107.0142 [astro-ph.CO]].

[9] M. S. Pshirkov, P. G. Tinyakov and F. R. Urban, arXiv:1504.06546 [astro-ph.CO].

[10] A. R. Taylor, J. M. Stil and C. Sunstrum, Astrophys. J. 702 (2009) 1230 doi:10.1088/0004$637 \mathrm{X} / 702 / 2 / 1230$.

[11] J. J. Condon, W. D. Cotton, E. W. Greisen, Q. F. Yin, R. A. Perley, G. B. Taylor and J. J. Broderick, Astron. J. 115 (1998) 1693. doi:10.1086/300337.

[12] A. M. Hammond, T. Robishaw and B. M. Gaensler, arXiv:1209.1438 [astro-ph.CO].

[13] D. H. F. M. Schnitzeler, Mon. Not. Roy. Astron. Soc. 409 (2010) L97 doi:10.1111/j.17453933.2010.00957.x [arXiv:1011.0737 [astro-ph.GA]].

[14] P. Coles and B. Jones, Mon. Not. Roy. Astron. Soc. 248 (1991) 1.

[15] H. Bi and A. F. Davidsen, Astrophys. J. 479 (1997) 523 doi:10.1086/303908 [astro-ph/9611062].

[16] L. Hollenstein, R. K. Jain and F. R. Urban, JCAP 1301 (2013) 013 doi:10.1088/14757516/2013/01/013 [arXiv:1208.6547 [astro-ph.CO]].

[17] P. G. Tinyakov and F. R. Urban, arXiv:1309.2270 [astro-ph.CO].

[18] P. A. R. Ade et al. [Planck Collaboration], arXiv:1502.01594 [astro-ph.CO].

[19] P. P. Kronberg, M. L. Bernet, F. Miniati, S. J. Lilly, M. B. Short and D. M. Higdon, Astrophys. J. 676 (2008) 7079 doi:10.1086/527281 [arXiv:0712.0435 [astro-ph]]. 